\title{
Clinical characteristics and treatment outcomes of mild to moderate COVID-19 patients in Saudi Arabia: A single centre study
}

\author{
Mohammad Albanghali ${ }^{1}$, Sirajudheen Anwar ${ }^{2}$, Mohammed Alzahrani ${ }^{3}$, Bassant Barakat ${ }^{1}$, \\ Abdul Haseeb ${ }^{4}$, and Saleh Alghamdi ${ }^{1}$
}

\author{
${ }^{1} \mathrm{Al}$ Baha University \\ ${ }^{2}$ University of Hail \\ ${ }^{3}$ King Fahad Hospital Al Baha \\ ${ }^{4}$ Umm Al-Qura University College of Pharmacy
}

April 28, 2021

\begin{abstract}
Background Since the severity of symptoms affects the treatment option for Coronavirus Disease 2019 (COVID-19) patients, the treatment pattern for mild to moderate non-ICU cases must be evaluated, particularly in the current scenario of mutation and variant strain for effective decision making. Objectives This retrospective study's objectives were to assess clinical and treatment outcomes in mild to moderate non-ICU COVID-19 patients in Saudi Arabia. Methods Non-ICU patients with COVID-19 with mild to moderate symptoms who were admitted to major tertiary care hospitals in Al Baha, Saudi Arabia, between April and August 2019 were studied retrospectively for clinical outcomes. Results A total of 811 people were admitted for COVID-19 treatment, with age ranging from 14 to 66 , diabetes mellitus $(31 \%, \mathrm{n}=248)$ and hypertension $(24 \%, \mathrm{n}=198)$ were the most common comorbid conditions. The majority of the patients $(77 \%, \mathrm{n}=621)$ had reported symptoms for COVID -19 infection. The hydroxychloroquine (HCQ) treated group (G1 $\mathrm{n}=466$ ) had an MD of 8 and an IQR of 5-13 for time in hospital with a 4.3 $\%$ mortality rate, while the non-HCQ group (G2 $\mathrm{n}=345)$ had an MD of 6 and an IQR of 3-11 for time in hospital with a 3.2 $\%$ mortality rate. The mortality rate among non-HCQ treated patients varied, but a combination of antiviral and antibiotic treatment was found to be effective, other most frequent intervention was analgesics $85.7 \%$, anticoagulant $75 \%$, minerals (Zinc $83 \%$ and Vit D3 $82 \%$ ). Conclusions The COVID 19 therapy and clinical outcomes from the past will be the guiding factor to treat the mutant strain infection in the future. Patients treated HCQ had a higher mortality rate, whereas those who were given a non-HCQ combination had a greater clinical outcome profile.
\end{abstract}

\section{Hosted file}

Main_manuscript.pdf available at https://authorea.com/users/410600/articles/519916-clinicalcharacteristics-and-treatment-outcomes-of-mild-to-moderate-covid-19-patients-in-saudiarabia-a-single-centre-study 